\title{
Upper bound on distance in the pants complex
}

\author{
Harriet Handel Moser
}

Received: 27 February 2013 / Accepted: 21 April 2013 / Published online: 17 May 2013

(C) Tbilisi Centre for Mathematical Sciences 2013

\begin{abstract}
The purpose of this paper is to establish an upper bound on the distance between two distinct pants decompositions in the pants complex. As part of the process, an upper bound on distance in the pants complex, modulo the action of the mapping class group, is also found. All of this is done by use of graph theory.
\end{abstract}

Keywords Pants complex · Pants decomposition · Distance · Graph · Upper bound Mathematics Subject Classification (2000) Primary 57M50; Secondary 57M15

\section{Introduction}

In 1980 Hatcher and Thurston [5] defined the pants graph, and showed that it is path connected. In this graph vertices are pants decompositions and an edge joins two vertices if there is an elementary move between the two corresponding pants decompositions. An elementary move occurs when one pants decomposition is transformed into another by replacing one curve with another one so that the two curves have minimal intersection (see Sect. 2). Distance comes from the path metric whereby the distance between two vertices joined by an edge is 1 . Approximately 20 years later, Hatcher et al. [4] defined the pants complex by adding 2-cells to the 1-dimensional pants graph with distance the same as in the 1-skeleton.

Communicated by Benson Farb.

Thanks to Joan Birman for her interest in and time devoted to discussions about this.

H. H. Moser $(\varangle)$

74 Wensley Drive, Great Neck, NY 11020, USA

e-mail: moser@math.columbia.edu 
The purpose of this paper is to establish an upper bound on the distance between two distinct pants decompositions in the pants complex. Graph theory is used to accomplish this. As part of the process, an upper bound on distance in the pants complex, modulo the action of the mapping class group, is found. A pants decomposition, $\mathbf{P}$, will have a pants decomposition graph where the vertices are the pairs of pants resulting from cutting the surface along the curves of $\mathbf{P}$, and the edges are the curves of $\mathbf{P}$. For a genus $g$ closed surface this is a trivalent graph on $2 g-2$ vertices with $3 g-3$ edges. An elementary move from $\mathbf{P}$ to another pants decomposition, $\mathbf{Q}$, induces a transformation from the pants decomposition graph for $\mathbf{P}$ to the pants decomposition graph for $\mathbf{Q}$, called an elementary shift. It can be shown that these two pants decomposition graphs are isomorphic if and only if the action of the mapping class group on the surface takes $\mathbf{P}$ to $\mathbf{Q}[8,11]$. Thus each orbit of this action will have a unique trivalent graph associated to it. The result is we can define a new graph, which we call the orbit graph, that is the pants graph modulo the mapping class group (see Fig. 4). In this graph, vertices are orbits of the action of the mapping class group on the pants graph and an edge corresponds to an elementary shift from the trivalent graph of one orbit to that of another orbit. The importance of the orbit graph is that a path in this graph from the orbit containing $\mathbf{P}$ to the orbit containing $\mathbf{Q}$ corresponds to a path of the same length from $\mathbf{P}$ to $\mathbf{Q}$ in the pants graph. Section 2 is devoted to a detailed discussion of several of the above mentioned graphs associated with pants decompositions. The concept of an orbit graph is new to this paper. In Sect. 3, principles of graph theory are applied to the orbits to find paths between orbits and their length. From now on, $\Sigma_{g}$ represents a closed surface of genus $g \geq 2$. Our first result, Theorem 1.1, gives an upper bound on the diameter of the orbit graph.

Theorem 1.1 Let $\mathbf{D}\left(\mathcal{O}\left(\Sigma_{g}\right)\right)$ denote the diameter of the orbit graph for $\Sigma_{g}$. Then

$$
\mathbf{D}\left(\mathcal{O}\left(\Sigma_{g}\right)\right) \leq \begin{cases}4 \log (g-1) !+4 g-6 & 2 \leq g \leq 5 \\ 4 \log (g-1) !+6 g-16 & 6 \leq g\end{cases}
$$

The literature has two other papers that derive an upper bound on the diameter of what we call the orbit graph. One is by Cavendish [2] and the other by Rafi and Tao [9]. They both concentrate on the asymptotic upper bound as genus approaches infinity. However, for any specific genus, both of these upper bounds are significantly larger than the one found by us in this paper. This is explained toward the end of Sect. 3, and demonstrated in a table. Neither of those papers address distance within an orbit or distance between two specific pants decompositions.

There is a special pants decomposition, $\mathbf{P}_{\text {loops }}$. This is seen, along with its pants decomposition graph, $\mathcal{P}_{\text {loops }}$, in Fig. 6. Section 4 finds a path between any two pants decompositions that are in $\mathcal{P}_{\text {loops }}$ and then calculates the length of the path. It makes use of a set of curves, $\left\{c_{1}, \ldots, c_{3 g-1}\right\}$, shown in Fig. 8, and twists about them, $\left\{T_{c_{1}}, \ldots, T_{c_{3 g-1}}\right\}$, which are a set of Lickorish generators for $\operatorname{Mod}\left(\Sigma_{g}\right)$. We call these curves the standard Lickorish generating curves and we refer to the twists as the standard Lickorish generators. We will denote two arbitrary pants decompositions on $\Sigma_{g}$ by $\mathbf{P}_{\mathbf{1}}$ and $\mathbf{P}_{\mathbf{2}}$ and the distance between them in the pants complex as $\mathbf{D}_{\mathbf{g}}\left(\mathbf{P}_{\mathbf{1}}, \mathbf{P}_{\mathbf{2}}\right)$. Our 
second result is Theorem 1.2, which finds an upper bound on distance in the pants complex.

Theorem 1.2 There is a pants decomposition, $\mathbf{P}_{\mathbf{l o o p s}}$, and two other pants decompositions, $\mathbf{R}_{\mathbf{1}}$ and $\mathbf{R}_{\mathbf{2}}$, in the same orbit as $\mathbf{P}_{\text {loops }}$ where $\mathbf{R}_{\mathbf{1}}$ (resp. $\mathbf{R}_{\mathbf{2}}$ ) is the end of a path from $\mathbf{P}_{\mathbf{1}}$ (resp. $\left.\mathbf{P}_{\mathbf{2}}\right)$. Let $w, h \in \operatorname{Mod}\left(\Sigma_{g}\right)$ such that $h\left(\mathbf{R}_{\mathbf{1}}\right)=\mathbf{P}_{\text {loops }}$ and $w\left(\mathbf{R}_{\mathbf{1}}\right)=\mathbf{R}_{\mathbf{2}}$. Then there are some standard Lickorish generating curves $\left\{c_{i_{1}}, \ldots, c_{i_{k}}\right\}$ such that $w=T_{h^{-1}\left(c_{i_{1}}\right)}^{e_{i_{1}}} \ldots T_{h^{-1}\left(c_{i_{k}}\right)}^{e_{i_{k}}}$ with $e_{i_{j}}= \pm 1$ for $1 \leq j \leq k$ where this is a minimum length word in these twists and

$$
\mathbf{D}_{\mathbf{g}}\left(\mathbf{P}_{\mathbf{1}}, \mathbf{P}_{2}\right) \leq\left\{\begin{array}{l}
4 \log (g-1) !+4 g-6+\sum_{1 \leq i_{j} \leq g}\left|e_{i_{j}}\right| \\
+4 \sum_{i_{j}=g+1,2 g-1}\left|e_{i_{j}}\right|+6 \sum_{g+2 \leq i_{j} \leq 2 g-2}\left|e_{i_{j}}\right| \quad 2 \leq g \leq 5 \\
4 \log (g-1) !+6 g-16+\sum_{1 \leq i_{j} \leq g}\left|e_{i_{j}}\right| \\
+4 \sum_{i_{j}=g+1,2 g-1}\left|e_{i_{j}}\right|+6 \sum_{g+2 \leq i_{j} \leq 2 g-2}\left|e_{i_{j}}\right| \quad 6 \leq g
\end{array}\right.
$$

We conclude with Sect. 5 .

\section{Graphs}

We begin with a few definitions. They can be found in the work of Margalit [6] and Putman [8], as well as many others. A pants decomposition is a maximal collection of pairwise disjoint isotopy classes of simple closed curves that are essential and nonperipheral. From now on, unless specified otherwise, the term curve will refer to the isotopy class of a curve. So for the surface $\Sigma_{g}$, a pants decomposition, $\mathbf{P}$, consists of $3 g-3$ curves that cut the surface into $2 g-2$ pairs of pants and is sometimes written as $\left\{c_{1}, \ldots, c_{3 g-3}\right\}$. If we consider $\left\{c_{1}, \ldots, c_{i-1}, c_{i+1}, \ldots, c_{3 g-3}\right\}$, these curves cut the surface so that all pieces are the same as before except for the pairs of pants containing $c_{i}$. This exception can be either a one-holed torus, $\mathcal{T}$, where $c_{i} \subset \mathcal{T}$, or a four-holed sphere where two pairs of pants have $c_{i}$ as a common curve separating them. An elementary move is a situation where the curve $c_{i}$ is replaced by another curve $c_{i}^{\prime}$ so that for the one-holed torus, $i\left(c_{i}, c_{i}^{\prime}\right)=1$, and for the four-holed sphere, $i\left(c_{i}, c_{i}^{\prime}\right)=2$. The result is another pants decomposition $\left\{c_{1}, \ldots, c_{i-1}, c_{i}^{\prime}, c_{i+1}, \ldots, c_{3 g-3}\right\}$. This can be seen in Fig. 1.

A pants graph, $\mathcal{P}\left(\Sigma_{g}\right)$, for $\Sigma_{g}$, is a 1-dimensional complex where vertices are pants decompositions and two vertices are joined by an edge when there is an elementary move between the two pants decompositions associated to the vertices. A path in the pants graph is from vertex to vertex along these edges. The length of a path is the number of edges traversed along the path and the distance between two vertices is the shortest path between them. Let $\mathbf{P}=\left\{c_{1}, \ldots, c_{3 g-3}\right\}$ be a pants decomposition for $\Sigma_{g}$. Then $\phi(\mathbf{P})$, the pants decomposition graph for $\mathbf{P}$, is a graph with $2 g-2$ vertices, one for each pair of pants obtained by cutting the surface along the curves of the decomposition, and $3 g-3$ edges, one for each curve in $\mathbf{P}$. A curve belonging to two 

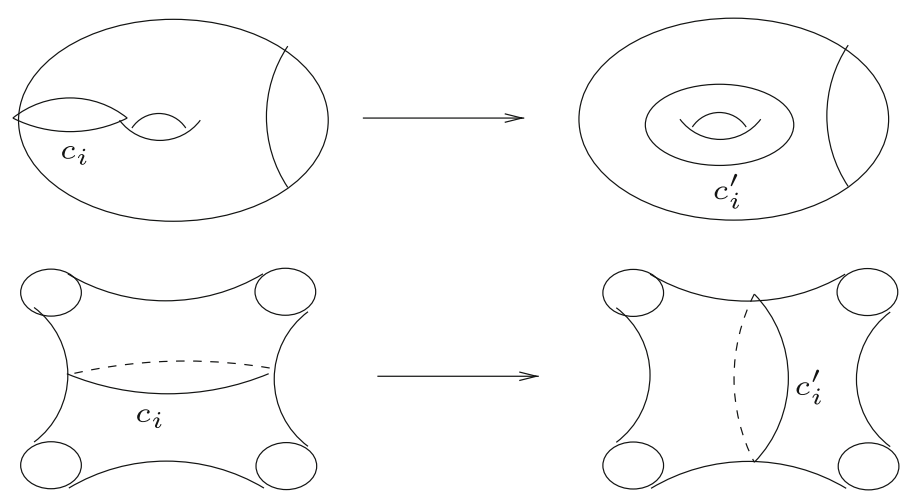

Fig. 1 Elementary moves

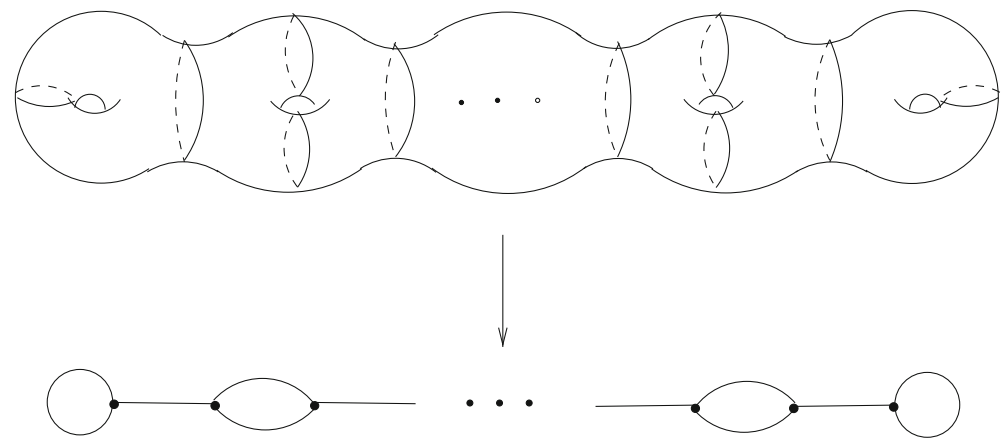

Fig. 2 Pants decomposition and pants decomposition graph

pairs of pants will correspond to an edge between the vertices for the pairs of pants, and a curve that is entirely in a pair of pants will correspond to a loop type edge at the vertex for that pair of pants. Figure 2 demonstrates this. The valence of every vertex will be 3 . This is true even when a loop is attached at a vertex since a loop has two arcs coming out of the vertex, and another edge goes to another vertex. Such a graph is called a trivalent or cubic graph.

There is a relationship between pants decomposition graphs that is similar to elementary moves between pants decompositions. Let there be an elementary move between the two pants decompositions $\mathbf{P}_{\mathbf{1}}=\left\{c_{1}, \ldots, c_{i-1}, c_{i}, c_{i+1}, \ldots, c_{3 g-3 g}\right\}$ and $\mathbf{P}_{\mathbf{2}}=\left\{c_{1}, \ldots, c_{i-1}, c_{i}^{\prime}, c_{i+1}, \ldots, c_{3 g-3}\right\}$, and $\phi\left(\mathbf{P}_{\mathbf{1}}\right)$ and $\phi\left(\mathbf{P}_{\mathbf{2}}\right)$ their corresponding pants decomposition graphs. If $i\left(c_{i}, c_{i}^{\prime}\right)=1$, then $c_{i}$ and $c_{i}^{\prime}$ are curves in the same one-holed torus, so they both occur as a loop attached to the same vertex while the rest of $\phi\left(\mathbf{P}_{\mathbf{1}}\right)$ and $\phi\left(\mathbf{P}_{\mathbf{2}}\right)$ remain unchanged and identical to each other. Therefore, $\phi\left(\mathbf{P}_{1}\right) \simeq \phi\left(\mathbf{P}_{\mathbf{2}}\right)$. Using Putman's method [8] for changing $\phi\left(\mathbf{P}_{\mathbf{1}}\right)$ to $\phi\left(\mathbf{P}_{2}\right)$ when $i\left(c_{i}, c_{i}^{\prime}\right)=2$, the following is done. In $\phi\left(\mathbf{P}_{\mathbf{1}}\right)$, the edge corresponding to $c_{i}$ will be adjacent to two vertices, $v_{1}$ and $v_{2}$. Collapse this edge so that $v_{1}$ and $v_{2}$ become one vertex. Four edges, two edges from each of the vertices $v_{1}$ and $v_{2}$, will now be adjacent to this resulting vertex. Now expand this vertex to two new vertices, with one edge 


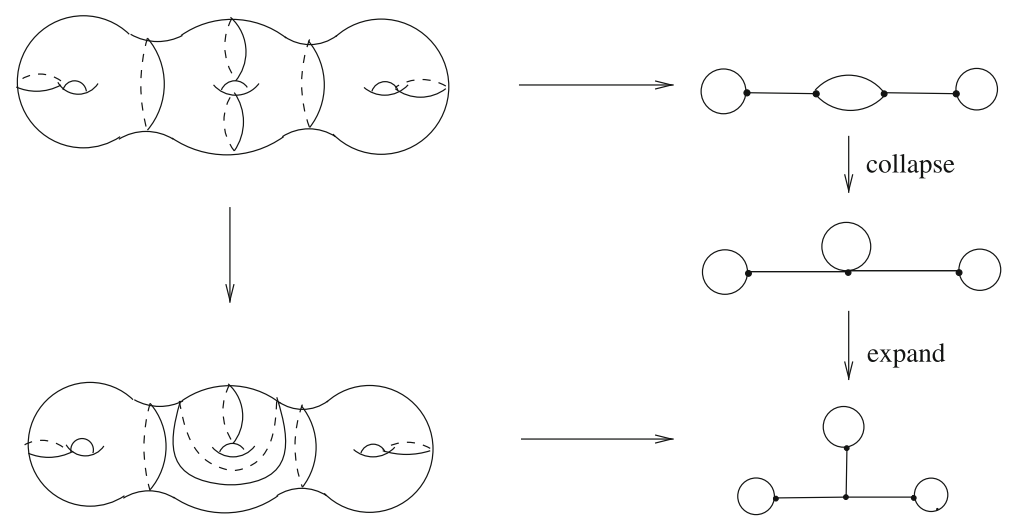

Fig. 3 Elementary shifts of pants decomposition graphs

between these two new vertices, and each new vertex will have one edge from $v_{1}$ and another edge from $v_{2}$. This transformation of $\phi\left(\mathbf{P}_{1}\right)$ is called an elementary shift. It can be seen in Fig. 3. Every elementary shift of $\phi\left(\mathbf{P}_{\mathbf{1}}\right)$ is the result of an elementary move of $\mathbf{P}_{\mathbf{1}}$.

A homeomorphism of a surface preserves intersection numbers, so a homeomorphism of $\Sigma_{g}$ takes a pants decomposition to another pants decomposition. Consequently, $\operatorname{Mod}\left(\Sigma_{g}\right)$, the mapping class group of $\Sigma_{g}$, operates on the pants graph, $\mathcal{P}\left(\Sigma_{g}\right)$. It has been shown that for any two pants decompositions $\mathbf{P}_{\mathbf{1}}$ and $\mathbf{P}_{\mathbf{2}}, \phi\left(\mathbf{P}_{\mathbf{1}}\right)$ and $\phi\left(\mathbf{P}_{\mathbf{2}}\right)$ are isomorphic if and only if there is an $f \in \operatorname{Mod}\left(\Sigma_{g}\right)$ such that $f \cdot \mathbf{P}_{\mathbf{1}}=\mathbf{P}_{\mathbf{2}}[8,11]$. Therefore, as the mapping class group acts on $\mathcal{P}\left(\Sigma_{g}\right)$, each orbit of vertices has a unique trivalent graph on $2 g-2$ vertices that is the pants decomposition graph, modulo isomorphism, of every pants decomposition in the orbit. From [11] we see that the orbit of an edge in $\mathcal{P}\left(\Sigma_{g}\right)$ between $\mathbf{P}_{\mathbf{1}}$ and $\mathbf{P}_{\mathbf{2}}$, under the action of the mapping class group, consists of all edges in $\mathcal{P}\left(\Sigma_{g}\right)$ that connect a pants decomposition in the orbit of $\mathbf{P}_{\mathbf{1}}$ to one in the orbit of $\mathbf{P}_{\mathbf{2}}$. Thus the orbit of the edge corresponds to an elementary shift between $\phi\left(\mathbf{P}_{1}\right)$ and $\phi\left(\mathbf{P}_{2}\right)$. This allows us to define a new graph, the orbit graph, $\mathcal{O}\left(\Sigma_{g}\right)$, where vertices are isomorphism classes of pants decomposition graphs and two vertices are joined by an edge if there is an elementary shift between the two corresponding pants decomposition graphs. We can now refer to a specific pants decomposition graph by the same term as the orbit corresponding to it under the action. Path and distance are defined as in all the other graphs discussed so far. In Fig. 4 we see the pants decomposition graphs for ten of the orbits for a genus 5 surface and a part of the orbit graph where the ten vertices are these orbits. Thus we see that the orbit graph is a graph of graphs.

The pants graph, $\mathcal{P}\left(\Sigma_{g}\right)$, is connected [5]. Therefore the orbit graph is connected, so there is a path connecting any two vertices in the orbit graph. Thus any pants decomposition graph can be transformed into any other one by a series of elementary shifts. Let $\mathbf{P}$ be a pants decomposition and $\mathcal{P}$ be it's orbit. Then, if $\mathcal{R}$ is another orbit that is one elementary shift away from $\mathcal{P}$, there will be some $\mathbf{R} \in \mathcal{R}$ that is one elementary move away from $\mathbf{P}$. Let $\mathbf{P}$ and $\mathbf{Q}$ be two pants decompositions, with 
1
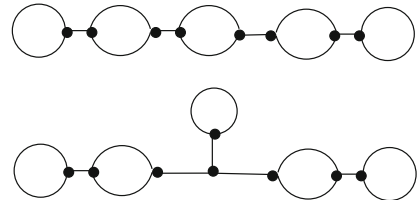

3
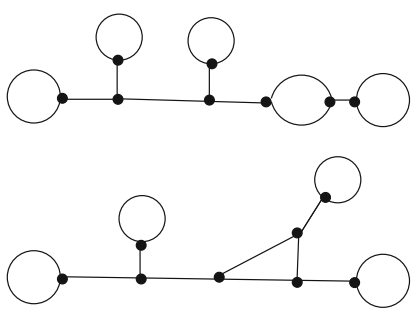

5

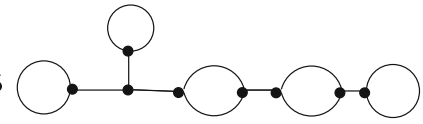

6

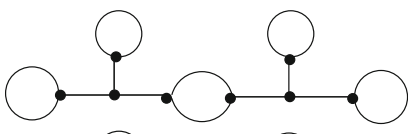

7

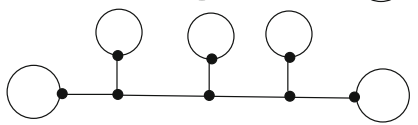

8

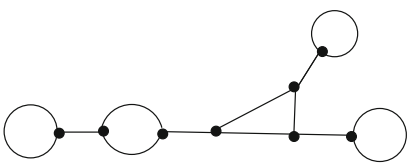

9

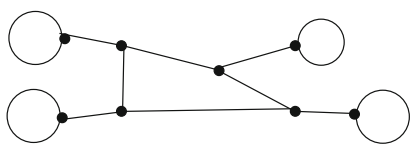

10

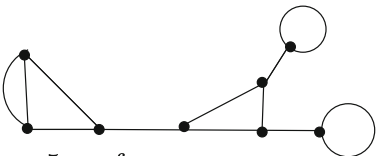

10 orbits for a genus 5 surface

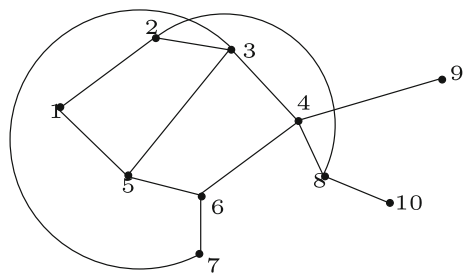

10 orbits in the orbit graph

Fig. 4 Orbits and the orbit graph

$\mathcal{P}$ and $\mathcal{Q}$ their orbits. Since there is a path from $\mathcal{P}$ to $\mathcal{Q}$ in the orbit graph, there will be a path in the pants graph from $\mathbf{P}$ to some pants decomposition $\mathbf{Q}^{\prime} \in \mathcal{Q}$, where each vertex in the pants graph path is a pants decomposition whose pants decomposition graph is an orbit corresponding to a vertex in the orbit path. Consequently, if we can determine distance in the orbit graph, it would remain to find distance between two pants decompositions in the same orbit.

\section{Distance in the orbit graph}

The girth of a graph is the length of the shortest cycle in the graph. This section shows how to transform a pants decomposition graph into another one with a loop, based on the girth of the graph. The calculation of an upper bound on girth enables us to find an upper bound on the number of elementary shifts needed to accomplish this transformation. Using this information we then show how to find the distance between two pants decomposition graphs. Graphs where all vertices have the same 
valence, like the trivalent pants decomposition graph for closed surfaces, has been the object of great interest to graph theorists. As early as 1967 Tutte [10] discussed these graphs and introduced the term $(k, G)$-cage. A $(k, G)$-cage is a graph with girth $G$ where all the vertices have valence $k$. The object of much of this study has been to see when such cages exist and how many vertices they have. Tutte [10] showed there is a graph whenever $k=3$, and also calculated the number of vertices for some specific instances of girth. However, for $G \geq 3$, there is the following general lower bound, $L B$, on vertex number for all cages $[1,10,12]$ :

$$
L B=\frac{2(k-1)^{G / 2}-2}{k-2} \text { for } G \text { even }
$$

and

$$
L B=\frac{k(k-1)^{\frac{G-1}{2}}-2}{k-2} \text { for } G \text { odd }
$$

Our pants decomposition graphs are $(3, G)$-cages, so for these graphs, where $G \geq 3$ :

$$
L B=2(2)^{G / 2}-2 \text { for } G \text { even }
$$

and

$$
L B=3(2)^{\frac{G-1}{2}}-2 \text { for } G \text { odd }
$$

Since these graphs all have $2 g-2$ vertices, their girth will be such that $L B \leq 2 g-2$. For $G \geq 3$ and even, we must have $G \geq 4$, so $G / 2 \geq 2$. Then $2^{G / 2} \geq 4>2$. Thus $2(2)^{G / 2}>(2)^{G / 2}+2$, so $L B>(2)^{G / 2}$. For $G$ odd,

$$
\begin{aligned}
L B & =3(2)^{\frac{G-1}{2}}-2 \\
& =\frac{3}{\sqrt{2}}(2)^{G / 2}-2 \\
& =\left(\frac{3}{\sqrt{2}}-1\right)(2)^{G / 2}-2+(2)^{G / 2} .
\end{aligned}
$$

For $G \geq 3,(2)^{G / 2} \geq(2)^{3 / 2}=2 \sqrt{2}$, so

$$
\begin{aligned}
\left(\frac{3}{\sqrt{2}}-1\right)(2)^{G / 2} & \geq\left(\frac{3}{\sqrt{2}}-1\right) 2 \sqrt{2} \\
& =6-2 \sqrt{2} \\
& >2 .
\end{aligned}
$$




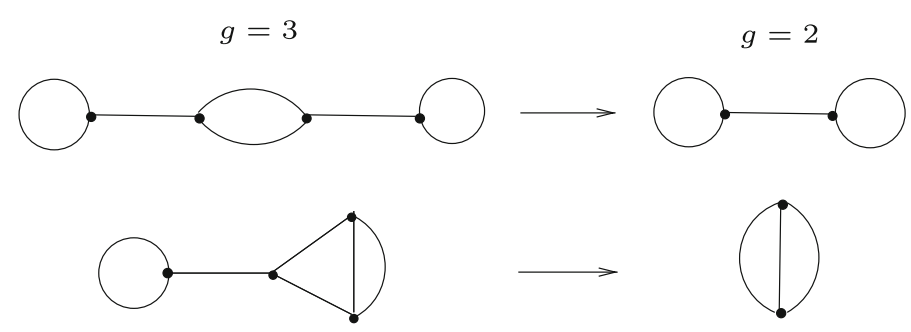

Fig. 5 Loop surgery

Therefore, $\left(\frac{3}{\sqrt{2}}-1\right)(2)^{G / 2}-2>0$, so

$$
L B=\left(\frac{3}{\sqrt{2}}-1\right)(2)^{G / 2}-2+(2)^{G / 2}>(2)^{G / 2} .
$$

Consequently, for any pants decomposition graph with girth $G \geq 3$, (2) $G / 2<2 g-2$. Taking $\operatorname{logs}$, we find $G / 2<\log (2 g-2)$. Then,

$$
\begin{aligned}
G & <2 \log 2(g-1) \\
& =2(1+\log (g-1)) .
\end{aligned}
$$

There are pants decomposition graphs that have girth of 2, such as the genus 2 orbit that does not have a loop (see Fig. 5). Since we are interested only in $g \geq 2,2(1+$ $\log (g-1)) \geq 2$, so $G \leq 2(1+\log (g-1))$ for all $G \geq 2$. Applying the "collapse and expand" process associated with an elementary shift (as seen in Fig. 3) to an edge of a cycle reduces the length of the cycle by 1 . So it would take one elementary shift less than the length of a cycle to reduce the cycle to a loop, and then $G-1$ elementary shifts would transform one pants decomposition graph, $\mathcal{P}$, to another one, $\mathcal{Q}$, which has a loop. Thus, the upper bound on the distance between two such graphs, $\mathcal{P}$ and $\mathcal{Q}$, in the orbit graph, would be $1+2 \log (g-1)$. Putman introduces a technique [8] which we call loop surgery. We can perform loop surgery on $\mathcal{Q}$ by cutting off the loop, the vertex the loop is attached to, and the edge adjacent to that vertex. The remaining vertex from that edge is then removed, leaving the other two edges that were attached to this last vertex unconnected to anything. Now join these two edges to make one smooth new edge in a new trivalent graph which has two less vertices (see Fig. 5). The result is a pants decomposition graph, $\overline{\mathcal{Q}}$, for a genus $g-1$ surface. Let $\mathbf{Y}$ denote the loop, the vertex the loop is attached to and the edge adjacent to that vertex.

Proposition 3.1 For each pants decomposition graph, $\mathcal{P}$, there is another pants decomposition graph, $\tilde{\mathcal{Q}}$, with g loops, where

$$
\mathbf{D}_{\text {orbit }_{\mathrm{g}}}(\mathcal{P}, \tilde{\mathcal{Q}}) \leq 2 g-3+2 \sum_{2 \leq i \leq g} \log (i-1) .
$$

Proof If $g=2$, it takes at most one elementary shift to transform $\mathcal{P}$ to a graph with two loops, so the proposition is true for $g=2$. Using induction, assume true for $g-1$. 
Let $g \geq 3$. Transform $\mathcal{P}$ to $\mathcal{Q}$ and perform loop surgery on $\mathcal{Q}$ to get $\overline{\mathcal{Q}}$ as described above. We now find a girth length cycle in $\overline{\mathcal{Q}}$ whose length will be less than or equal to $2+2 \log (g-2)$. Then $\overline{\mathcal{Q}}$ can be transformed to a graph, $\overline{\mathcal{Q} \mathcal{Q}}$, with a loop, by doing at most $1+2 \log (g-2)$ elementary shifts along all but one of the edges of the cycle. This corresponds to a path in $\mathcal{O}\left(\Sigma_{g-1}\right)$ of length $1+2 \log (g-2)$. We now lift this path to $\mathcal{O}\left(\Sigma_{g}\right)$ by reattaching $\mathbf{Y}$ to $\overline{\mathcal{Q}}$ to get $\mathcal{Q}$ and carrying $\mathbf{Y}$ along with the transformations. If $g=3, \overline{\mathcal{Q}}$ will be the genus 2 graph with two loops, so it is possible that in it's lift $\mathbf{Y}$ will be attached to one of these loops. In this case, one more elementary shift is required to move $\mathbf{Y}$ to the edge between these two loops. Thus it is true for $g=3$. Now let $g \geq 4$. If none of the edges of the cycle in $\overline{\mathcal{Q}}$ are the result of joining the two loose edges of $\mathcal{P}$ after the removal of $\mathbf{Y}$, then the end result of the lift is that $1+2 \log (g-2)$ elementary shifts will give us a graph in $\mathcal{O}\left(\Sigma_{g}\right)$ that is the lift of $\overline{\mathcal{Q}}$ and it has one more loop than $\mathcal{Q}$ has. Otherwise, another vertex gets added to the cycle in $\overline{\mathcal{Q}}$ at the point where $\mathbf{Y}$ is attached, so one more elementary shift must be done to get this new loop. So we see that it takes at most $1+2 \log (g-1)+1+2 \log (g-2)+1$ elementary shifts to get a graph with two more loops than $\mathcal{P}$ has. The logic is that we can continue the process recursively by pulling off one new loop for each succeeding lower genus, going all the way down to $g=2$. This is formalized by an induction argument. By induction we know that it takes at most $2(g-1)-3+2 \sum_{2 \leq i \leq g-1} \log (i-1)$ elementary shifts to convert $\overline{\mathcal{Q}}$ to a graph with $g-1$ loops. The path of this transformation can be lifted to $\mathcal{O}\left(\Sigma_{g}\right)$, starting at $\mathcal{Q}$. It takes at most $1+2 \log (g-1)$ elementary shifts to convert $\mathcal{P}$ to $\mathcal{Q}$. Finally, if in the lift $\mathbf{Y}$ is attached to the transformation of $\overline{\mathcal{Q}}$ along a loop edge, the attaching vertex of $\mathbf{Y}$ adds a vertex to the loop, creating a cycle of length 2 and keeping the number of loops in the lift of $\overline{\mathcal{Q}}$ at $g-1$. One more elementary shift is needed to convert the cycle to another loop, giving us a graph with $g$ loops. Denote this graph by $\tilde{\mathcal{Q}}$ (see Fig. 7). Then

$$
\begin{aligned}
\mathbf{D}_{\text {orbit }_{\mathbf{g}}}(\mathcal{P}, \tilde{\mathcal{Q}}) & \leq 1+2 \log (g-1)+2(g-1)-3+2 \sum_{2 \leq i \leq g-1} \log (i-1)+1 \\
& \leq 2 g-3+2 \sum_{2 \leq i \leq g} \log (i-1)
\end{aligned}
$$

So it is true for all $g \geq 2$.

We now want to find the distance from $\tilde{\mathcal{Q}}$ to $\mathcal{P}_{\text {loops }}$, a special trivalent graph on $2 g-2$ vertices with $g$ loops, as in Fig. 6. We do this because we know how to calculate distance between pants decompositions in the orbit $\mathcal{P}_{\text {loops }}$. Our graphs are isomorphism classes of graphs, so when $2 \leq g \leq 5$ we have $\tilde{\mathcal{Q}}$ isomorphic to $\mathcal{P}_{\text {loops }}$, resulting in $\mathbf{D}_{\text {orbit }}\left(\tilde{\mathcal{Q}}, \mathcal{P}_{\text {loops }}\right)=0$. For $g \geq 6$, a graph like $\tilde{\mathcal{Q}}$ would look like the one in Fig. 7. It has $n$ branches, with $l_{i}+1$ loops on the $i$ th branch for $1 \leq i \leq n$. An elementary shift according to the "collapse and expand" process on the edge from vertex $v_{i}$ to vertex $v_{i, 1}$ transforms the graph by moving the loop at the vertex $v_{i, 1}$ to the edge between $v_{i-1}$ and a new vertex at the base of the $i$ th branch and shortening the $i$ th branch by 1 loop. So it would take $l_{i}$ elementary shifts to flatten the entire branch. Therefore it would 

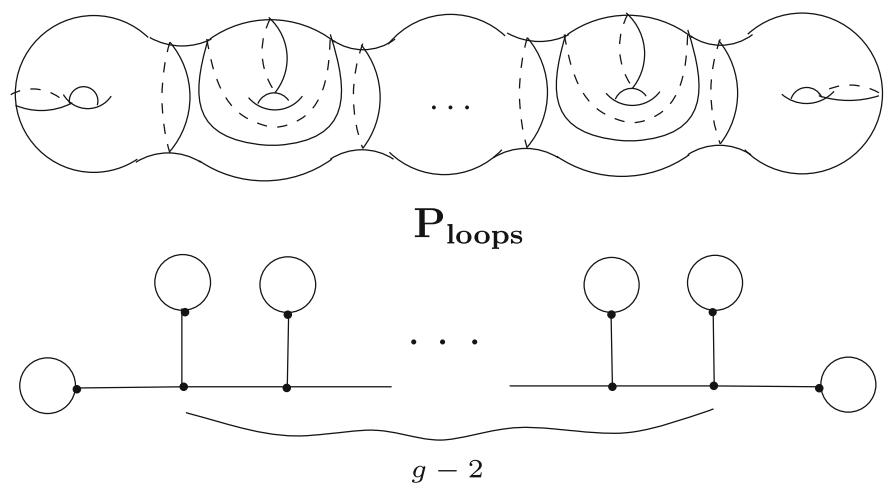

$\mathcal{P}_{\text {loops }}$

Fig. 6 Special pants decomposition and pants decomposition graph with $g$ loops

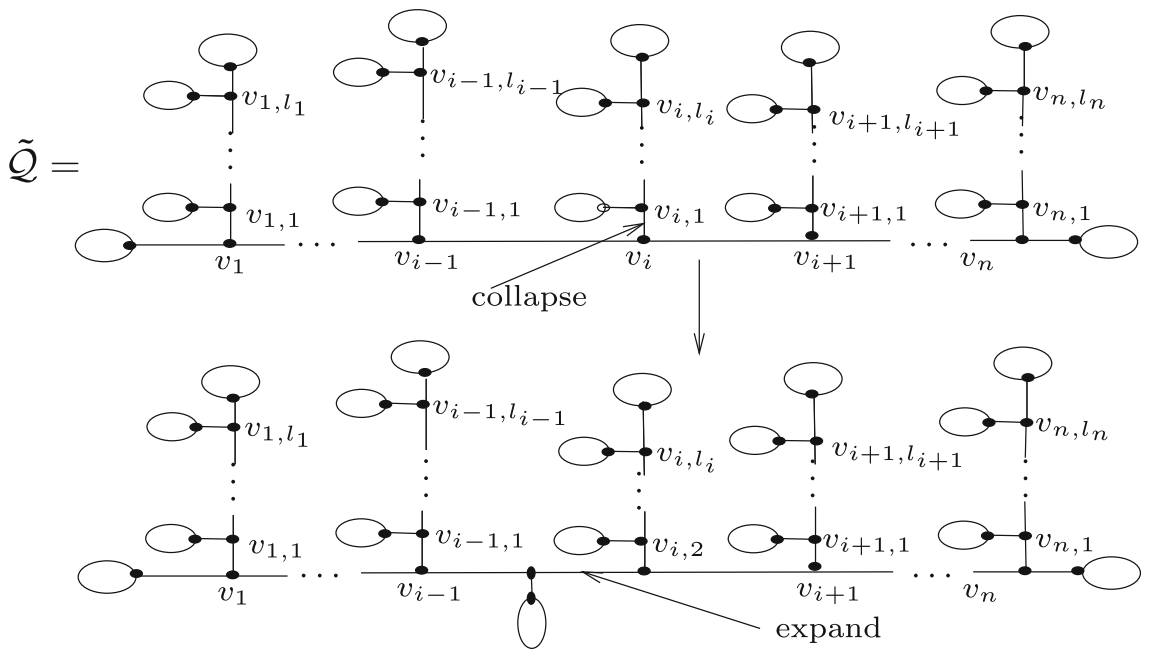

Fig. 7 Move the loop on $\tilde{\mathcal{Q}}$

take $\sum_{1 \leq i \leq n} l_{i}$ elementary shifts to convert $\tilde{\mathcal{Q}}$ to $\mathcal{P}_{\text {loops }}$. However, there are $l_{i}+1$ loops on each branch vertex $v_{1}$ to $v_{n}$, so $g=$ number of loops $=n+2+\sum_{1 \leq i \leq n} l_{i}$. If $n$ is equal to either 1 or $2, \tilde{\mathcal{Q}}$ is isomorphic to $\mathcal{P}_{\text {loops }}$, so $\mathbf{D}_{\text {orbitg }}\left(\tilde{\mathcal{Q}}, \mathcal{P}_{\text {loops }}\right)=0$. Then if $n \geq 3, \sum_{1 \leq i \leq n} l_{i} \leq g-5$. Thus

$$
\mathbf{D}_{\text {orbitg }}\left(\tilde{\mathcal{Q}}, \mathcal{P}_{\text {loops }}\right) \leq \max (0, g-5)
$$

Proof of Theorem 1.1 Let $\mathcal{P}_{\mathbf{1}}$ and $\mathcal{P}_{\mathbf{2}}$ denote the pants decomposition graphs for $\mathbf{P}_{1}$ and $\mathbf{P}_{2}$, respectively, and $\tilde{\mathcal{Q}}_{1}$ and $\tilde{\mathcal{Q}}_{2}$ the respective transformations of $\mathcal{P}_{\mathbf{1}}$ and $\mathcal{P}_{\mathbf{2}}$ to graphs with $g$ loops. Then 
Table 1 Comparison of upper bounds

\begin{tabular}{llll}
\hline Genus & Moser & Cavendish & Rafi-Tao \\
\hline 2 & 2.000 & 46.0000 & 40.1027 \\
3 & 10.0000 & 96.0000 & 118.9648 \\
4 & 20.3398 & 148.7188 & 209.4618 \\
5 & 32.3398 & 204.0000 & 300.5137 \\
6 & 47.6275 & 261.4525 & 409.0029 \\
10 & 117.8765 & 507.5940 & 863.3850 \\
20 & 331.0218 & $1,193.6684$ & $2,183.2609$ \\
50 & $1,118.2571$ & $3,539.8839$ & $6,889.1641$ \\
100 & $2,656.4845$ & $7,897.4852$ & $15,961.7874$ \\
200 & $6,134.9466$ & $17,412.5993$ & $36,267.2371$ \\
500 & $18,017.5508$ & $48,845.5840$ & $104,659.6478$ \\
750 & $28,794.3573$ & $76,786.9314$ & $167,101.4012$ \\
1000 & $40,061.7288$ & $105,708.7269$ & $230,945.6910$ \\
5000 & $246,865.0744$ & $621,490.9524$ & $1,412,410.0690$ \\
20000 & $1,147,562.1146$ & $2,806,016.4388$ & $6,513,851.3966$ \\
49000 & $3,064,966.7632$ & $7,381,536.1568$ & $17,298,782.5923$ \\
\hline
\end{tabular}

$$
\begin{aligned}
\mathbf{D}_{\text {orbit }_{\mathbf{g}}}\left(\mathcal{P}_{\mathbf{1}}, \mathcal{P}_{\mathbf{2}}\right) \leq & \mathbf{D}_{\text {orbit }_{\mathbf{g}}}\left(\mathcal{P}_{\mathbf{1}}, \tilde{\mathcal{Q}}_{\mathbf{1}}\right)+\mathbf{D}_{\text {orbit }_{\mathbf{g}}}\left(\tilde{\mathcal{Q}}_{\mathbf{1}}, \mathcal{P}_{\text {loops }}\right) \\
& +\mathbf{D}_{\text {orbit }_{\mathbf{g}}}\left(\mathcal{P}_{\mathbf{2}}, \tilde{\mathcal{Q}}_{\mathbf{2}}\right)+\mathbf{D}_{\text {orbit }_{\mathbf{g}}}\left(\tilde{\mathcal{Q}}_{\mathbf{2}}, \mathcal{P}_{\text {loops }}\right) \\
\leq & 2\left(2 g-3+2 \sum_{2 \leq i \leq g} \log (i-1)+\max (0, g-5)\right) \\
\leq & \left\{\begin{array}{cl}
4 g-6+4 \sum_{2 \leq i \leq g} \log (i-1) & 2 \leq g \leq 5 \\
6 g-16+4 \sum_{2 \leq i \leq g} \log (i-1) & 6 \leq g
\end{array}\right.
\end{aligned}
$$

The diameter of the orbit graph for $\Sigma_{g}$ is the upper bound on distance between any two vertices in this graph. We know this exists because there are only a finite number of distinct trivalent graphs that have a given even number of vertices. Since $\mathbf{P}_{\mathbf{1}}$ and $\mathbf{P}_{\mathbf{2}}$ are arbitrary,

$$
\mathbf{D}\left(\mathcal{O}\left(\Sigma_{g}\right)\right) \leq \begin{cases}4 \log (g-1) !+4 g-6 & 2 \leq g \leq 5 \\ 4 \log (g-1) !+6 g-16 & 6 \leq g\end{cases}
$$

Both Cavendish [2] and Rafi and Tao [9] have calculated an upper bound on the diameter of what we call the orbit graph. Below is an explanation and statement of these two different bounds. We show that both of these upper bounds are larger than the one derived by us in this paper. Table 1 further demonstrates this by comparing some of these values with ours. Cavendish has shown that for a cubic graph with $n$ vertices: 
(1) it takes $2 n+2$ Whitehead moves to transform the graph to a second cubic graph on $n$ vertices whose spanning tree has only valence 1 and valence 3 vertices, (2) another $2 n \log (2 n)+4 \log (2 n)$ moves, at most, will transform this second graph to a third cubic graph with the same spanning tree, where all valence 1 vertices of the spanning tree will have a loop, (3) this last type of graph can be treated as a tree when considering Whitehead moves and (4) any two spanning trees on $n$ vertices are at most $n$ moves apart when both trees have only valence 1 and valence 3 vertices [2]. Consequently, any two cubic graphs on $n$ vertices will be at most $2(2 n+2+2 n \log (2 n)+4 \log (2 n))+n$ moves apart. Letting $n=2 g-2$, we have $26 g+8 g \log (g-1)-6$ as the Cavendish upper bound on the diameter of the space of trivalent graphs on $2 g-2$ vertices before extracting the leading term to find the asymptotic upper bound with respect to genus.

Rafi and Tao [9] define a new metric of simultaneous Whitehead moves on the same space of cubic graphs with $n=2 g-2$ vertices, and find an upper bound on the distance between any two such cubic graphs in terms of this new metric. Multiplying this distance by $g$ will give us the Rafi-Tao upper bound on the diameter of the space of trivalent graphs on $2 g-2$ vertices because at most $g$ Whitehead moves will produce the same result as one simultaneous Whitehead move. The asymptotic upper bound comes from this value. Rafi and Tao do this by identifying a family of trees where each graph has an associated tree and if two such trees differ by simultaneous Whitehead moves, then their associated graphs will differ by the same number of simultaneous Whitehead moves. Then they show: (1) it takes at $\operatorname{most} \log _{6 / 5}(n+1)$ simultaneous Whitehead moves to transform the height of a tree so that the new height, $h$, will be at most $6 \log n$ and (2) it takes at most $h-3+d$ moves to transform a tree of height at most $6 \log n$ to a special tree called a "fully sorted tree" on $n$ vertices, where $d=\lceil\log n\rceil$. Thus two cubic graphs are at most $2\left(\log _{6 / 5}(n+1)+6 \log n-3+\lceil\log n\rceil\right)$ simultaneous Whitehead moves apart. Replacing $n$ by $2 g-2$ and multiplying by $g$, we have $2 g \log _{6 / 5}(2 g-1)+12 g \log (g-1)+8 g+2 g\lceil\log (g-1)\rceil$ as the Rafi-Tao upper bound on the diameter of the space of trivalent graphs on $2 g-2$ vertices before extracting the leading term to find the asymptotic upper bound with respect to genus.

Lemma 3.2 For any specific genus, g, the upper bound on the diameter of the orbit graph derived by us is smaller than the upper bounds found by either Cavendish or Rafi and Tao.

Proof For $1 \leq i \leq g-1$ and $g \geq 2, \log i \leq \log (g-1)$, so

$$
\begin{aligned}
4 \log (g-1) ! & =4 \sum_{i=1}^{g-1} \log i \\
& \leq 4 \sum_{i=1}^{g-1} \log (g-1) \\
& =4(g-1) \log (g-1) \\
& \leq 8 g \log (g-1) \\
& \leq 12 g \log (g-1)
\end{aligned}
$$




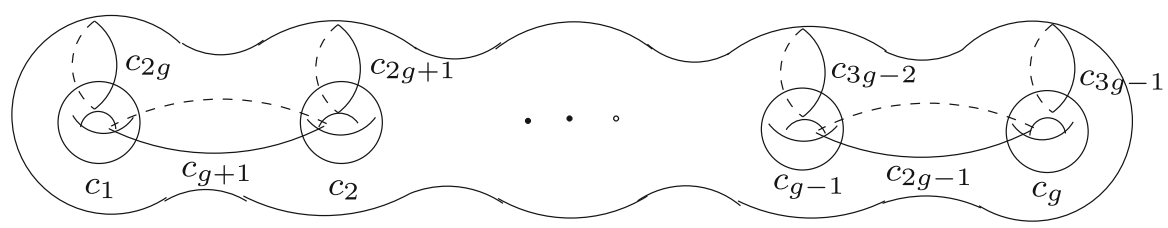

Fig. 8 Standard Lickorish generating curves

For $2 \leq g \leq 5,4 g-6$ is less than both $26 g-6$ and $2 g \log _{6 / 5}(2 g-1)+8 g+$ $2 g\lceil\log (g-1)\rceil$, so combining this with Eqs. 3 and 4 we see that our lemma is true for $2 \leq g \leq 5$

Similarly, for $g \geq 6,6 g-16$ is less than both $26 g-6$ and $2 g \log _{6 / 5}(2 g-1)+$ $8 g+2 g\lceil\log (g-1)\rceil$, so our lemma is true for $g \geq 6$.

The process of finding an upper bound on the distance between $\mathbf{P}_{\mathbf{1}}$ and $\mathbf{P}_{\mathbf{2}}$ involves constructing a pair of paths in the orbit graph from $\mathcal{P}_{\mathbf{1}}$ and $\mathcal{P}_{\mathbf{2}}$ to $\mathcal{P}_{\text {loops }}$ and calculating an upper bound on their length. The paths will have corresponding paths of the same length in the pants graph from $\mathbf{P}_{\mathbf{1}}$ (resp. $\mathbf{P}_{\mathbf{2}}$ ) to some $\mathbf{R}_{\mathbf{1}}$ (resp. $\mathbf{R}_{\mathbf{2}}$ ) in $\mathcal{P}_{\text {loops }}$. Since

$$
\mathbf{D}_{\mathbf{g}}\left(\mathbf{P}_{\mathbf{1}}, \mathbf{P}_{\mathbf{2}}\right) \leq \mathbf{D}_{\mathbf{g}}\left(\mathbf{P}_{\mathbf{1}}, \mathbf{R}_{\mathbf{1}}\right)+\mathbf{D}_{\mathbf{g}}\left(\mathbf{P}_{\mathbf{2}}, \mathbf{R}_{\mathbf{2}}\right)+\mathbf{D}_{\mathbf{g}}\left(\mathbf{R}_{\mathbf{1}}, \mathbf{R}_{\mathbf{2}}\right)
$$

we have

$$
\mathbf{D}_{\mathbf{g}}\left(\mathbf{P}_{\mathbf{1}}, \mathbf{P}_{\mathbf{2}}\right) \leq \begin{cases}4 \log (g-1) !+4 g-6+\mathbf{D}_{\mathbf{g}}\left(\mathbf{R}_{\mathbf{1}}, \mathbf{R}_{\mathbf{2}}\right) & 2 \leq g \leq 5 \\ 4 \log (g-1) !+6 g-16+\mathbf{D}_{\mathbf{g}}\left(\mathbf{R}_{\mathbf{1}}, \mathbf{R}_{\mathbf{2}}\right) & 6 \leq g\end{cases}
$$

\section{Distance within the orbit $\mathcal{P}_{\text {loops }}$}

Let $\left\{c_{1}, \ldots, c_{3 g-1}\right\}$ be the standard Lickorish generating curves, with the corresponding standard Lickorish generators being $\left\{T_{c_{1}}, \ldots, T_{c_{3 g-1}}\right\}$ (see Fig. 8). There is a path in the pants graph from $\mathbf{P}_{\text {loops }}$ to both $T_{c_{i}}\left(\mathbf{P}_{\text {loops }}\right)$ and $T_{c_{i}}^{-1}\left(\mathbf{P}_{\text {loops }}\right)$ of length $l_{c_{i}}$ where:

$$
l_{c_{i}}= \begin{cases}1 & 1 \leq i \leq g \\ 4 & i=g+1,2 g-1 \\ 6 & g+2 \leq i \leq 2 g-2 \\ 0 & 2 g \leq i \leq 3 g-1\end{cases}
$$

These paths can be seen in Fig. 9, where only the piece of $\mathbf{P}_{\text {loops }}$ that changes in the pants decompositions along the path from $\mathbf{P}_{\text {loops }}$ to $T_{c_{i}}$ ( $\left.\mathbf{P}_{\text {loops }}\right)$ is shown. Putman has constructed paths from another pants decomposition that is in a different orbit. They obviously differ from ours, though they inspired this construction [8]. We now use our path lengths as follows.

Lemma 4.1 Let $w \in \operatorname{Mod}\left(\Sigma_{g}\right)$, so there are some standard Lickorish generating curves $\left\{c_{i_{1}}, \ldots, c_{i_{k}}\right\}$ such that $w=T_{c_{i_{1}}}^{e_{i_{1}}} \ldots T_{c_{i_{k}}}^{e_{i_{k}}}$ with $e_{i_{j}}= \pm 1$ for $1 \leq j \leq k$. Then 


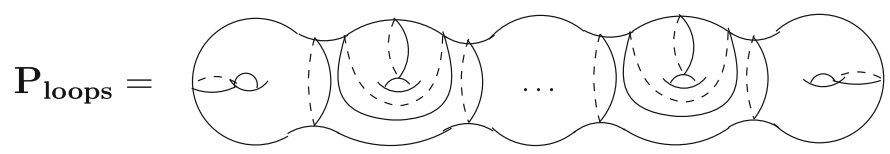

1 Elementary Shift Takes $\mathbf{P}_{\text {loops }}$ to $T_{c_{i}}\left(\mathbf{P}_{\text {loops }}\right)$ for $1 \leqslant i \leqslant g$

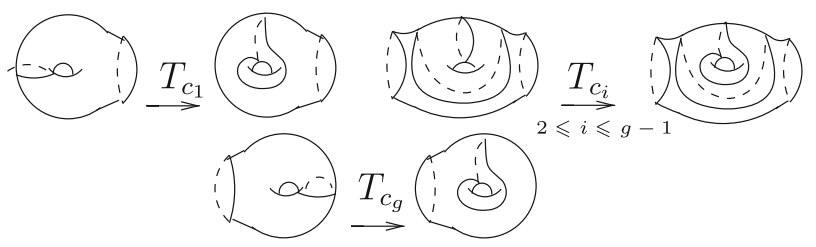

4 Elementary Shifts Take $\mathbf{P}_{\text {loops }}$ to $T_{c_{g+1}}\left(\mathbf{P}_{\text {loops }}\right)$

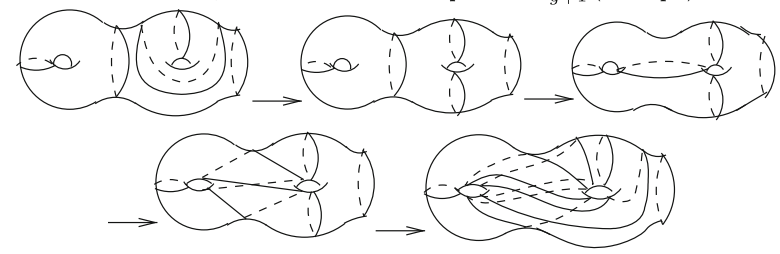

6 Elementary Shifts Take $\mathbf{P}_{\text {loops }}$ to $T_{c_{i}}\left(\mathbf{P}_{\text {loops }}\right)$ for $g+2 \leqslant i \leqslant 2 g-2$

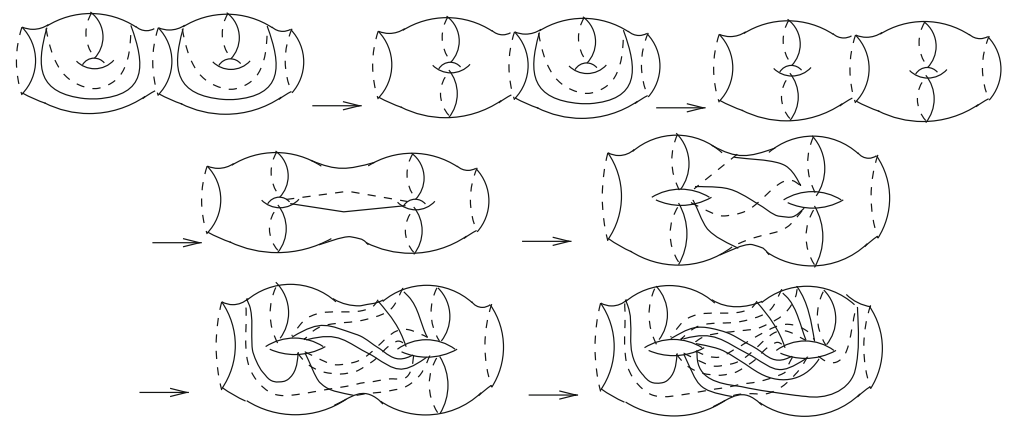

4 Elementary Shifts Take $\mathbf{P}_{\text {loops }}$ to $T_{c_{2 g-1}}\left(\mathbf{P}_{\text {loops }}\right)$

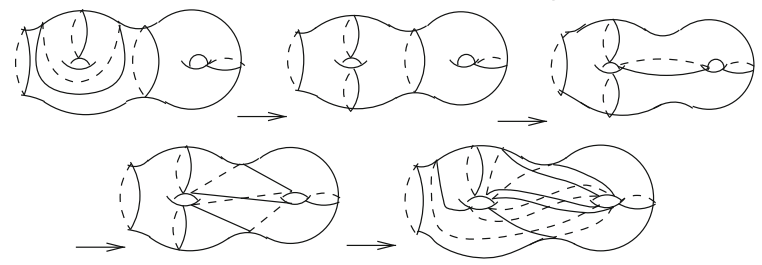

Fig. 9 Paths from $\mathbf{P}_{\text {loops }}$

there is a path from $\mathbf{P}_{\text {loops }}$ to $w$ (P. $\left.\mathbf{P}_{\text {loops }}\right)$ of length equal to $\sum_{j=1}^{k} l_{c_{i_{j}}}$, where $l_{c_{i_{j}}}$ is the length of a path from $\mathbf{P}_{\text {loops }}$ to both $T_{c_{i_{j}}}\left(\mathbf{P}_{\text {loops }}\right)$ and $T_{c_{i_{j}}}^{-1}\left(\mathbf{P}_{\text {loops }}\right)$.

Proof Consider the path from $\mathbf{P}_{\text {loops }}$ to $T_{c{ }_{j}}^{e_{i}}\left(\mathbf{P}_{\text {loops }}\right)$. Let $f \in \operatorname{Mod}\left(\Sigma_{g}\right)$. Apply $f$ to every pants decomposition in the path. Since intersection numbers are preserved, we 
get a path from $f\left(\mathbf{P}_{\text {loops }}\right)$ to $f\left(T_{c_{i} j}^{e_{i}}\left(\mathbf{P}_{\text {loops }}\right)\right)=f T_{c_{i_{j}}}^{e_{i}}\left(\mathbf{P}_{\text {loops }}\right)$ that is the same length, $l_{c_{i_{j}}}$, as the path from $\mathbf{P}_{\text {loops }}$ to $T_{c_{i_{j}}}^{e_{i_{j}}}$ ( $\left.\mathbf{P}_{\text {loops }}\right)$. Consequently, there will be a path from

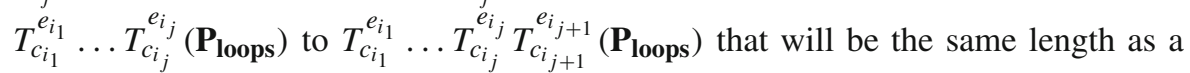
path from $\mathbf{P}_{\text {loops }}$ to $T_{c_{i_{j+1}}}^{e_{i j+1}}\left(\mathbf{P}_{\text {loops }}\right)$. Thus we have a path

$$
\begin{gathered}
\mathbf{P}_{\text {loops }} \rightarrow T_{c_{i_{1}}}^{e_{i_{1}}}\left(\mathbf{P}_{\text {loops }}\right) \rightarrow T_{c_{i_{1}}}^{e_{i_{1}}}\left(T_{c_{i_{2}}}^{e_{i_{2}}}\left(\mathbf{P}_{\text {loops }}\right)\right) \\
\rightarrow \cdots \rightarrow T_{c_{i_{1}}}^{e_{i_{1}}} \ldots T_{c_{i_{k-1}}}^{e_{i_{k-1}}}\left(T_{c_{i_{k}}}^{e_{i_{k}}}\left(\mathbf{P}_{\text {loops }}\right)\right)
\end{gathered}
$$

whose length is $\sum_{j=1}^{k} l_{c_{i_{j}}}$. Since $w=T_{c_{i_{1}}}^{e_{i_{1}}} \ldots T_{c_{i_{k-1}}}^{e_{i_{k-1}}} T_{c_{i_{k}}}^{e_{i_{k}}}$, we have a path from $\mathbf{P}_{\text {loops }}$ to $w$ (P. $\left.\mathbf{P}_{\text {loops }}\right)$ whose length is $\sum_{j=1}^{k} l_{c_{i_{j}}}$.

Lemma 4.2 Let $\left\{T_{c_{1}}, \ldots, T_{c_{3 g-1}}\right\}$ be the standard Lickorish generators. Then for any $f \in \operatorname{Mod}\left(\Sigma_{g}\right),\left\{T_{f\left(c_{1}\right)}, \ldots, T_{f\left(c_{3 g-1}\right)}\right\}$ is another set of Lickorish generators for the mapping class group.

Proof Let $h \in \operatorname{Mod}\left(\Sigma_{g}\right)$. Then $f^{-1} h f \in \operatorname{Mod}\left(\Sigma_{g}\right)$. Therefore there are some standard Lickorish generating curves $\left\{c_{i_{1}}, \ldots, c_{i_{k}}\right\}$ such that $f^{-1} h f=T_{c_{i_{1}}}^{e_{i_{1}}} \ldots T_{c_{i_{k}}}^{e_{i_{k}}}$ with $e_{i_{j}}= \pm 1$ for $1 \leq j \leq k$. It is a basic fact about Dehn twists that $T_{f(a)}=f T_{a} f^{-1}$ for all $f \in \operatorname{Mod}\left(\Sigma_{g}\right)$ [3], so $f^{-1} T_{f\left(c_{i_{j}}\right)}^{e_{i_{j}}} f=T_{c_{i_{j}}}^{e_{i_{j}}}$ for $1 \leq j \leq k$. Thus,

$$
\begin{aligned}
f^{-1} h f & =f^{-1} T_{f\left(c_{i_{1}}\right)}^{e_{i_{1}}} f f^{-1} T_{f\left(c_{i_{2}}\right)}^{e_{i_{2}}} f \ldots f^{-1} T_{f\left(c_{i_{k}}\right)}^{e_{i_{k}}} f \\
& =f^{-1} T_{f\left(c_{i_{1}}\right)}^{e_{i_{1}}} T_{f\left(c_{i_{2}}\right)}^{e_{i_{2}}} \ldots T_{f\left(c_{i_{k}}\right)}^{e_{i_{k}}} f .
\end{aligned}
$$

Hence, $h=T_{f\left(c_{i_{1}}\right)}^{e_{i_{1}}} T_{f\left(c_{i_{2}}\right)}^{e_{i_{2}}} \ldots T_{f\left(c_{i_{k}}\right)}^{e_{i_{k}}}$, so $\left\{T_{f\left(c_{1}\right)}, \ldots, T_{f\left(c_{3 g-1}\right)}\right\}$ generates $\operatorname{Mod}\left(\Sigma_{g}\right)$.

Proposition 4.3 Let $\mathbf{R}_{\mathbf{1}}, \mathbf{R}_{\mathbf{2}} \in \mathcal{P}_{\text {loops }}$ and $w, h \in \operatorname{Mod}\left(\Sigma_{g}\right)$ such that $h\left(\mathbf{R}_{\mathbf{1}}\right)=\mathbf{P}_{\text {loops }}$ and $w\left(\mathbf{R}_{\mathbf{1}}\right)=\mathbf{R}_{\mathbf{2}}$. For some subset of the standard Lickorish generators, $\left\{c_{i_{1}}, \ldots, c_{i_{k}}\right\}$, we have $w=T_{h^{-1}\left(c_{i_{1}}\right)}^{e_{i_{1}}} \ldots T_{h^{-1}\left(c_{i_{k}}\right)}^{e_{i_{k}}}$, with $e_{i_{j}}= \pm 1$ for $1 \leq j \leq k$, where this is a minimum length word in these twists. There is a path from $\mathbf{R}_{\mathbf{1}}$ to $\mathbf{R}_{\mathbf{2}}$ in $\mathcal{P}_{\text {loops }}$ of length $\sum_{1 \leq i_{j} \leq g}\left|e_{i_{j}}\right|+4 \sum_{i_{j}=g+1,2 g-1}\left|e_{i_{j}}\right|+6 \sum_{g+2 \leq i_{j} \leq 2 g-2}\left|e_{i_{j}}\right|$.

Proof By Lemma 4.2, $\left\{T_{h^{-1}\left(c_{1}\right)}, \ldots, T_{h^{-1}\left(c_{3 g-1}\right)}\right\}$ also generates $\operatorname{Mod}\left(\Sigma_{g}\right)$, so $w$ can be expressed in terms of these generators. We can assume that $w$ is a minimal length word in these generators since Mosher [7] has shown that the mapping class group of closed surfaces is automatic, ensuring that the word problem is solvable for it. Since $h\left(\mathbf{R}_{\mathbf{1}}\right), h\left(\mathbf{R}_{\mathbf{2}}\right) \in \mathcal{P}_{\text {loops }}$, there must be some $f \in \operatorname{Mod}\left(\Sigma_{g}\right)$ whereby $f\left(h\left(\mathbf{R}_{\mathbf{1}}\right)\right)=$ $h\left(\mathbf{R}_{\mathbf{2}}\right)$. Then

$$
\begin{aligned}
f\left(h\left(\mathbf{R}_{\mathbf{1}}\right)\right) & =h\left(w\left(\mathbf{R}_{\mathbf{1}}\right)\right) \\
& =h\left(T_{h^{-1}\left(c_{i_{1}}\right)}^{e_{i_{1}}} \ldots T_{h^{-1}\left(c_{i_{k}}\right)}^{e_{i_{k}}}\left(\mathbf{R}_{\mathbf{1}}\right)\right)
\end{aligned}
$$




$$
\begin{aligned}
& =T_{h h^{-1}\left(c_{i_{1}}\right)}^{e_{i_{1}}} \ldots T_{h h^{-1}\left(c_{i_{k}}\right)}^{e_{i_{k}}}\left(h\left(\mathbf{R}_{\mathbf{1}}\right)\right) \\
& =T_{c_{i_{1}}}^{e_{i_{1}}} \ldots T_{c_{i_{k}}}^{e_{i_{k}}}\left(h\left(\mathbf{R}_{\mathbf{1}}\right)\right),
\end{aligned}
$$

so $T_{c_{i_{1}}}^{e_{i_{1}}} \ldots T_{c_{i_{k}}}^{e_{i_{k}}}$ is a word in the standard Lickorish generators that takes $\mathbf{P}_{\text {loops }}$ to $h\left(\mathbf{R}_{\mathbf{2}}\right)$. Lemma 4.1 tells us there is a path from $\mathbf{P}_{\text {loops }}$ to $h\left(\mathbf{R}_{\mathbf{2}}\right)$ of length $\sum_{j=1}^{k} l_{c_{i_{j}}}$. Substitute the values for $l_{c_{i_{j}}}$ in Eq. 6, to get a path of length equal to $\sum_{1 \leq i_{j} \leq g}\left|e_{i_{j}}\right|+4 \sum_{i_{j}=g+1,2 g-1}\left|e_{i_{j}}\right|+6 \sum_{g+2 \leq i_{j} \leq 2 g-2}\left|e_{i_{j}}\right|$. We now apply $h^{-1}$ to every pants decomposition in this path to get a path of the same length from $h^{-1}\left(\mathbf{P}_{\text {loops }}\right)=h^{-1}\left(h\left(\mathbf{R}_{\mathbf{1}}\right)\right)=\mathbf{R}_{\mathbf{1}}$ to $h^{-1}\left(h\left(\mathbf{R}_{\mathbf{2}}\right)\right)=\mathbf{R}_{\mathbf{2}}$.

Proof of Theorem 1.2 From our work on orbits we know that corresponding to an orbit path from $\mathcal{P}_{\mathbf{1}}$ (resp. $\mathcal{P}_{\mathbf{2}}$ ) to $\mathcal{P}_{\text {loops }}$ will be a path of the same length in the pants graph from $\mathbf{P}_{\mathbf{1}}$ (resp. $\mathbf{P}_{\mathbf{2}}$ ) to some $\mathbf{R}_{\mathbf{1}}$ (resp. $\mathbf{R}_{\mathbf{2}}$ ) in $\mathcal{P}_{\text {loops }}$. Let $w, h \in \operatorname{Mod}\left(\Sigma_{g}\right)$ where $w \mathbf{R}_{\mathbf{1}}=\mathbf{R}_{\mathbf{2}}$ and $h \mathbf{R}_{\mathbf{1}}=\mathbf{P}_{\text {loops }}$. By Proposition 4.3, there is some subset of the standard Lickorish generators, $\left\{c_{i_{1}}, \ldots, c_{i_{k}}\right\}$, such that $w=T_{h^{-1}\left(c_{i_{1}}\right)}^{e_{i_{1}}} \ldots T_{h^{-1}\left(c_{i_{k}}\right)}^{e_{i_{k}}}$, with $e_{i_{j}}=$ \pm 1 for $1 \leq j \leq k$, where this is a minimum length word in these twists and there is a path from $\mathbf{R}_{\mathbf{1}}$ to $\mathbf{R}_{\mathbf{2}}$ in $\mathcal{P}_{\text {loops }}$ of length equal to $\sum_{1 \leq i_{j} \leq g}\left|e_{i_{j}}\right|+4 \sum_{i_{j}=g+1,2 g-1}\left|e_{i_{j}}\right|+$ $6 \sum_{g+2 \leq i j \leq 2 g-2}\left|e_{i_{j}}\right|$. This plus Eq. 5 gives us

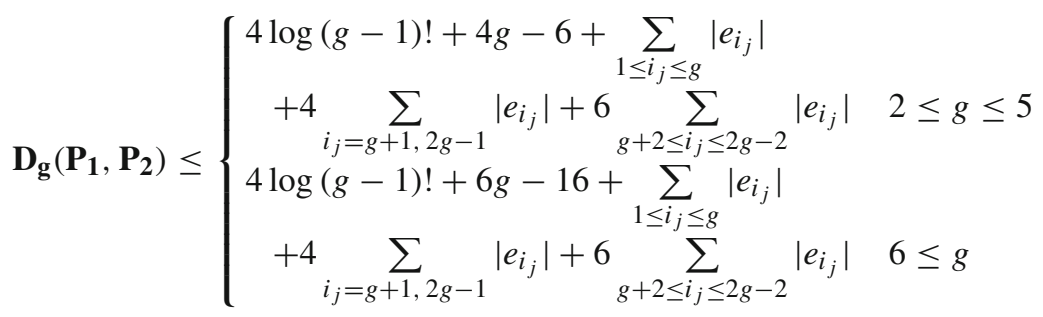

\section{Open problems and limitations}

It would be interesting to investigate the structure of the orbit graph. If we could document the length of a path between any two elements of any orbit, so as not to have to go through $\mathcal{P}_{\text {loops }}$, we might have a shorter path and thus a better upper bound. We also don't know if the shortest word operating on one pants decomposition, taking it to another, will yield the shortest path between these two pants decompositions. It would be nice to extend this work to surfaces with boundaries.

\section{References}

1. Bollobas, B.: External Graph Theory. New York Academic Press, Toronto (1978)

2. Cavendish, W.: Growth of the diameter of the pants graph modulo the mapping class group (2009). http://www.math.princeton.edu/ wcavendi/ 
3. Farb, B., Margolit, D.: A Primer on Mapping Class Groups. Princeton Mathematical, Princeton (2011)

4. Hatcher, A., Lochak, P., Schneps, L.: On the Teichmuller tower of mapping class groups. J. Reine Angew. Math. 521, 1-24 (2000)

5. Hatcher, A., Thurston, W.: A presentation for the mapping class group of a closed orientable surface. Topology 19, 221-237 (1980)

6. Margalit, D.: Automorphisms of the pants complex. Duke Math. J. 121(3), 457-479 (2004)

7. Mosher, L.: Mapping class groups are automatic. Math. Res. Lett. 1, 249-255 (1994)

8. Putman, A.: A note on the connectivity of certain complexes associated to surfaces. L'Enseignment Mathematique Journal 54(2), 287-301 (2008)

9. Rafi, K., Tao, J.: The diameter of the thick part of modulii space and simultaneous whitehead moves (2011). arXiv:math.GT/1108.4150

10. Tutte, W.T.: Connectivity in Graphs. Toronto University Press, Toronto (1967)

11. Wolf, U.: The action of the mapping class group on the pants complex (2009). http://www.math.kit. edu/iag3/ wolf/media/wolf-the-action-of-the-mapping-class-group-on-the-pants-complex

12. Wong, P.K.: Cages-a survey. J. Graph Theory 6, 1-22 (1982) 